

Ingeniería. Investigación y Tecnología

ISSN: 1405-7743

iit.revista@gmail.com

Universidad Nacional Autónoma de México

México

Torchia-Núñez, J.C.; Porta-Gándara, M.A.; Cervantes-de Gortari, J.G.

Análisis de exergía en estado permanente de un destilador solar simple Ingeniería. Investigación y Tecnología, vol. XI, núm. 1, 2010, pp. 25-34

Universidad Nacional Autónoma de México

Distrito Federal, México

Disponible en: http://www.redalyc.org/articulo.oa?id=40415986004

- Cómo citar el artículo

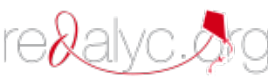

- Número completo

- Más información del artículo

- Página de la revista en redalyc.org

Sistema de Información Científica

Red de Revistas Científicas de América Latina, el Caribe, España y Portugal Proyecto académico sin fines de lucro, desarrollado bajo la iniciativa de acceso abierto 


\title{
Análisis de exergía en estado permanente de un destilador solar simple
}

\section{Steady-State Exergy Analysis of a Simple Solar Still}

\author{
J.C. Torchia-Núñez \\ Departamento de Termoenergía, Facultad de Ingeniería, \\ Universidad Nacional Autónoma de México. \\ E-mail:maporta@cibnor.mx \\ M.A. Porta-Gándara \\ Centro de Investigaciones Biológicas del Noroeste, S.C. \\ La Paz, B.C.S. \\ E-mail:tadeo_isidoro_cruz@yahoo.com \\ J.G. Cervantes-de Gortari \\ Departamento de Termoenergía, Facultad de Ingeniería, \\ Universidad Nacional Autónoma de México. \\ E-mail: (autor para correspondencia):jgonzalo@servidor.unam.mx
}

(Recibido: febrero de 2007; aceptado: mayo de 2009)

\section{Resumen}

En este trabajo se presenta un análisis teórico de exergía de un destilador solar simple en estado permanente. Mediante un balance de energía sobre los tres componentes principales de un destilador solar -colector, salmuera y cubierta de vidrio- para un conjunto de valores de parámetros -irradiancia solar, temperatura ambiente y espesor de aislamiento-, se obtienen las temperaturas de los componentes para distintas condiciones. Con estas temperaturas se pueden encontrar los flujos de exergía y eficiencias de segunda ley en un destilador solar. Los resultados muestran que la irradiancia solar es el parámetro más influyente en los procesos de transporte dentro del destilador, seguido por el espesor del aislamiento térmico. Para una irradiancia solar de $1,000 \mathrm{~W} / \mathrm{m}^{2}$, el colector cede $13 \%$ del total de exergía que llega al agua salada. El agua salada utiliza más del $6 \%$ de este total para la evaporación. Las irreversibilidades alcanzan $86 \%$ de la exergía total. La relación energía/exergía muestra que el componente más eficiente en el destilador solar es la masa de agua salada con más de $90 \%$ para cualquier valor de los parámetros estudiados mientras que el colector alcanza el $23 \%$ para un valor de $1,000 \mathrm{~W} / \mathrm{m}^{2}$ de irradiancia solar. El destilador solar es un dispositivo que aprovecha el calentamiento de un colector con alta eficiencia y la discusión sobre su uso, como todos los sistemas solares, no debe ser por sus limitaciones termodinámicas.

Descriptores: exergía, eficiencia, destiladorsolar, irreversibilidades, termodinámica.

\section{Abstract}

This paper presents a steady-state, theoretical exergy analysis of a solar still, focused on the exergy flows in the components of the still: collector plate, brine and glass cover. The analytical approach states an energy balance for each component resulting in three coupled equations where three parameters-solar irradiance, ambient temperature and 
insulation thickness-are studied. The energy balances are solved to find temperatures of each component; these temperatures are used to compute energy and exergy flows. Results in the steady-state regime show that the collector transports $13 \%$ of incident radiation exergy to the heat the brine, while in the brine the evaporation exergy accounts for the 6\% of total exergy. Exergy/energy ratio shows that the most efficient component is the brine reaching more than $90 \%$ for almost any value of the parameters under study, while the collector has a $23 \%$ exergy/energy ratio for a 1,000 W/ $\mathrm{m}^{2}$ solar energy input. The solar still is a device that uses efficiently the heating of a collector and the general discussion about its promotion should not, as it must be with almost every other solar device, be about thermodynamic limitations.

Keywords: Exergy, efficiency, solar still, irreversibilities, thermodynamics.

\section{Introducción}

Los destiladores solares simples se utilizan comúnmente en zonas costeras áridas para la obtención de agua fresca de bajo costo a partir del agua de mar. El diseño más simple de un destilador solar consiste en una caja rectangular con una cubierta de vidrio transparente como se muestra en la figura 1.

El proceso de destilación solar es el siguiente:

a) el destilador se llena parcialmente con salmuera en el fondo del depósito, que es una superficie negra y rugosa (colector) utilizada para absorber la radiación incidente que cruza la cubierta de vidrio y la masa de agua;

b) el colector incrementa su temperatura y transfiere calor al agua;

c) ocurre evaporación de salmuera en la superficie libre;

d) se produce un flujo de convección natural de aire húmedo dentro del destilador debido a la diferencia de temperaturas entre la superficie libre de la salmuera caliente y la cubierta de vidrio a menor temperatura;

e) esta cubierta inclinada sirve como placa condensadora donde se forma el agua destilada que fluye por gravedad hacia un pequeño canal recolector en la pared lateral menos alta del arreglo.

Se pueden lograr cerca de 5 litros $/ \mathrm{m}^{2}$ diarios de agua destilada en La Paz, México (24 de latitud norte) en un día de verano, haciendo al proceso económicamente atractivo para aquéllas áreas donde existe escasez de agua potable. Estudios económicos de destilación apuntan a que la destilación solar es viable cuando la producción no excede los $200 \mathrm{~m}^{3}$ de destilado al día (Malik et al., 1982).

El fenómeno de destilación solar es un proceso inestable donde se tiene evaporación, convección de calor, masa y condensación. Una descripción detallada del equipo y su operación puede encontrarse en Porta et al. (1997).

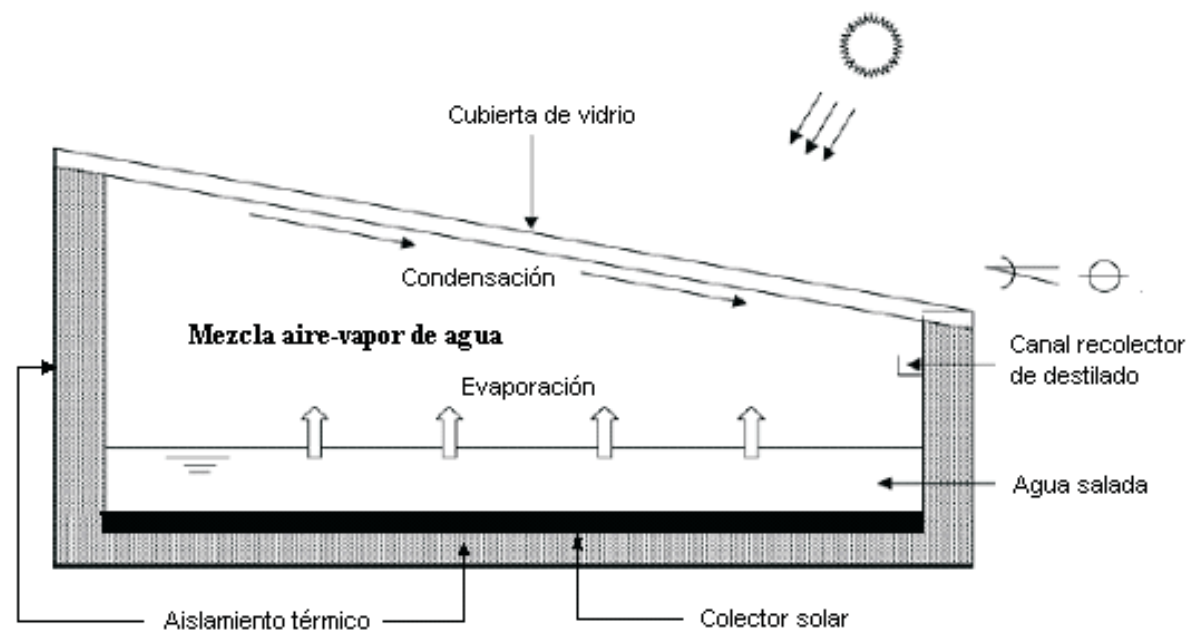

Figura 1. Esquema de un destilador solar 
Aunque el modelado matemático de un destilador solar se encuentra en gran parte desarrollado, existen recientes estudios en el área (Hongfei et al., 2002; Kumar y Tiwari, 1996; Shukla y Sorayan, 2005; Tiwari et al., 2003; Tripathi y Tiwari, 2005), en los cuales se busca mejorar las relaciones de transferencia de calor al tomar en cuenta todos los parámetros, condiciones ambientales y configuraciones geométricas posibles. La mayoría de los modelos en destilación solar están basados en un análisis de parámetros concentrados donde se consideran tres componentes principales (Malik et al., 1982):

1. El colector solar, que actúa tanto como recipiente de agua de mar como la superficie que absorbe la radiación solar.

2. El agua salada.

3. La cubierta de vidrio donde condensa el vapor de agua.

El resultado es un sistema de tres ecuaciones acopladas que describen el comportamiento térmico de los tres componentes del destilador. Las variables desconocidas de estas ecuaciones son la temperatura del colector, de la salmuera y la cubierta de vidrio. Con estas temperaturas, se calculan los flujos de exergía y la destrucción de exergía. La mayoría de los estudios teóricos en destilación solar se concentran en la resolución de un balance de energía para obtener las temperaturas, los flujos de calor y la cantidad de destilado producido.

El estudio de los sistemas solares, de acuerdo con las leyes de la termodinámica, han llamado la atención de varios investigadores, cuyos estudios han sido ampliamente discutidos y revisados por Bejan (1987) y Petela (2003). El análisis termodinámico es una herramienta efectiva para la obtención de información precisa sobre la eficiencia y pérdidas, debido a irreversibilidades en los procesos más complejos. Es claro que la tendencia actual en el diseño de procesos reales es la minimización de producción de entropía, con el fin de diseñar sistemas que sean óptimos técnica y económicamente.

Aunque los métodos que emplean a la segunda ley y el concepto de exergía están bien establecidas (Bejan, 1982; Gaggioli, 1998; Kotas, 1994; Petela, 1964), las herramientas utilizadas en la ingeniería solar aún se basan en la primera ley de la Termodinámica. La primera ley se refiere únicamente a la conservación de energís y no proporciona información sobre cómo, dónde y en qué cantidad se degrada el funcionamiento del sistema. El análisis de exergía es una herramienta poderosa en el diseño, optimización y evaluación del funcionamiento de los sistemas de energía. Hasta donde los autores conocen, no existen resultados previamente publicados de análisis de segunda ley en destiladores solares excepto por el trabajo hecho por Kwatra (1996). En este artículo se presenta un análisis de los flujos y destrucción de exergía en un destilador solar simple operando en régimen permanente. Para el modelo se supone lo siguiente:

- La mezcla aire-vapor de agua y el aislamiento no se consideran sistemas o medios participantes.

- El agua de mar y la cubierta de vidrio no interactúan con la radiación solar incidente.

- El valor del factor de forma en el intercambio de radiación entre el colector y la cubierta de vidrio es 1.

- El destilador solar es un sistema cerrado.

- Las propiedades físicas de los materiales no son afectadas por cambios en la temperatura.

- No existen gradientes de temperatura en los componentes del destilador.

- La cantidad de agua en el destilador es constante.

\section{Flujos de energía, exergía e irreversibilidades en un destilador solar}

La exergía $B_{S}$ contenida en la radiación isotrópica del Sol, considerado como cuerpo negro, a la temperatura de su superficie $T_{S}$, referida a la temperatura ambiente $T_{a}$ (Petela, 1964) es:

$$
B_{S}=G_{S}\left(1+\frac{1}{3}\left(\frac{T_{a}}{T_{S}}\right)^{4}-\frac{4 T_{a}}{3 T_{S}}\right),
$$

donde $G_{S}$ es la irradiancia solar i.e. la radiación instantánea por unidad de área que alcanza una superficie horizontal tomando en cuenta el ángulo sólido entre la Tierra y el Sol, y la variación cenital a lo largo del día.

La exergía asociada a una interacción de calor, que no sea radiación, puede expresarse según:

$$
B=q\left(1-\frac{T_{a}}{T}\right),
$$

donde $q$ es el flujo de calor, y $T$ la temperatura del sistema.

Considere el colector solar en estado estacionario sujeto a flujos de energía y exergía (figura 2).

Se pueden expresar los balances de energía y exergía, respectivamente, según: 
$\varepsilon_{c o l} G_{S}=q_{w}+q_{\text {ins }}$

$$
\varepsilon_{c o l} B_{S}=I_{c o l}+B_{w}+B_{i n s} .
$$

En la ecuación 3, el primer término del lado derecho $\varepsilon_{c o l} G_{S}$, es la energía absorbida en el colector que llega del sol, $q_{w}$ es el calor que cede el colector al agua salada, y $q_{\text {ins }}$, las pérdidas de calor hacia el medio ambiente, a través del aislamiento térmico.

La ecuación 4, por su parte, expresa el balance de exergía en el colector, donde $\varepsilon_{c o l} B_{S}$ es la exergía que absorbe el colector de la radicación solar incidente, $I_{c o l}$ las irreversibilidades producidas en el colector, $B_{w}$ la exergía contenida en el calor que fluye del colector al agua sala$\mathrm{da}, \mathrm{y} B_{i n s}$ las pérdidas de exergía a través del aislamiento.
Por otra parte, las figuras 3 y 4 muestran los flujos de energía y exergía en los dos componentes restantes del destilador solar bajo análisis: agua salada y cubierta de vidrio, respectivamente.

De la misma manera que con el colector, se pueden escribir los balances de energía y exergía para el agua salada y la cubierta de vidrio, respectivamente:

$$
\begin{aligned}
& q_{w}=q_{w g} \\
& B_{w}=B_{w g}+I_{w} \\
& q_{w g}=q_{a} \\
& B_{w g}=B_{a}+I_{g} .
\end{aligned}
$$

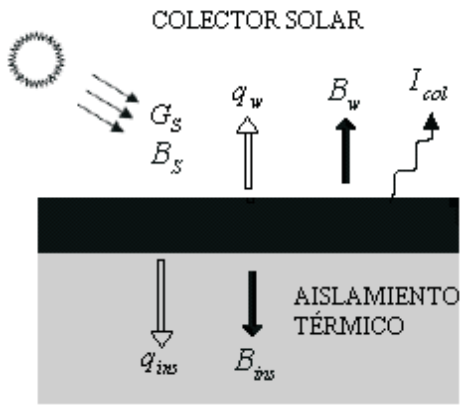

Figura 2. Flujos de energía y exergía en el colector solar

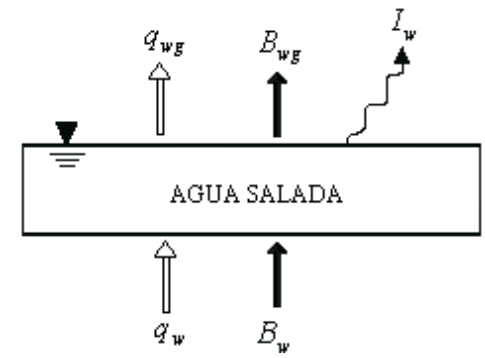

Figura 3. Flujos de energía y exergía en el agua salada

CUBIERTA DE VIDRIO

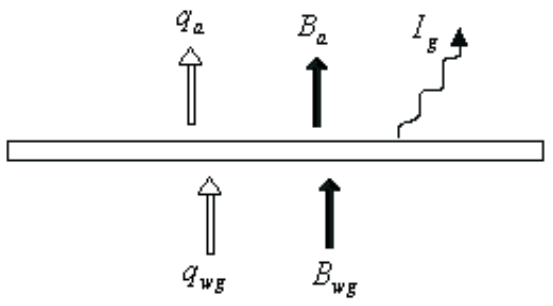

Figura 4. Flujos de energía y exergía en la cubierta de vidrio 
Las ecuaciones (5) y (6) son los balances de energía y exergía para el agua salada, donde $q_{w g}$ y $B_{w g}$ son el calor y la exergía total, respectivamente, transferidos por convección, evaporación y radiación desde la superficie libre hasta el interior del vidrio. Los balances de energía y exergía en la cubierta de vidrio se presentan en las ecs. (7) y (8), respectivamente, donde $q_{a}$ es el calor transferido por convección forzada y radiación hacia el medio ambiente y $B_{a}$ la exergía asociada a $q_{a}$. En las ecuaciones (6) y (8), $I_{w}$ y $I_{c o l}$ son las irreversibilidades en el agua salada y en el colector. Los flujos de exergía, que no son transportados por radiación, se calculan usando la ecuación (2). Los flujos de exergía de radiación se calculan según la ecuación (1). Para los cálculos de exergía térmica se toma como temperatura de referencia la temperatura del medio ambiente $T_{a}$. Los flujos de calor calcularon según las conocidas relaciones de Dunkle (Malik et al. 1982). Con esta información podemos definir las eficiencias energéticas (de primera ley) y de exergía (de segunda ley) para el colector y el agua salada.

Las eficiencias de primera ley se pueden escribir como:

$$
\begin{aligned}
& \eta_{e n, c o l}=\frac{q_{w}}{G_{S}} \\
& \eta_{e n, e v}=\frac{q_{e v}}{q_{w}},
\end{aligned}
$$

para el colector y el agua salada, respectivamente. En la ec. (10) $q_{e v}$ es el calor de evaporación que fluye entre el agua salada y el vidrio. Para poder definir la eficiencia de segunda ley utilizando la exergía, se debe definir una relación producto/recurso (salida/entrada) en términos de exergías, dependiendo del propósito del sistema o el proceso. Kwatra encontró una expresión para la eficiencia de segunda ley que relaciona la producción de destilado con la entrada de exergía. Sin embargo, es posible expresar la eficiencia de segunda ley como una relación entre salida/entrada de exergías en el colector de la siguiente manera:

$$
\eta_{e x, c o l}=\frac{B_{w}}{\varepsilon_{c o l} B_{S}}
$$

Esta es la relación de entrada de exergía desde el colector utilizada para calentar el agua salada y la exergía incidente del sol absorbida por el colector, $\varepsilon_{\text {col }} B_{S}$. Para el agua salada (figura 3 ), podemos también expresar una eficiencia de segunda ley, como se muestra:

$$
\eta_{e x, e v}=\frac{B_{e v}}{B_{w}},
$$

donde la exergía de evaporación $B_{e v}$ es el efecto útil y el recurso es la exergía transferida por el colector hacia el agua salada, $B_{w}$. Este análisis supone que la exergía útil global del proceso es la exergía de evaporación, i.e. todo el vapor de agua se condensa y el proceso de condensación y recolección no producen irreversibilidades. Por lo tanto, no se define una eficiencia de segunda ley para la cubierta de vidrio.

Las ecuaciones de energía en estado permanente se resolvieron para distintas condiciones reflejadas en los valores de los parámetros. Cuando un parámetro varía, los demás permanecían constantes.

En la tabla 1 se muestran, junto con otros datos empleados en el cálculo, el valor de los parámetros cuando no variaban.

\section{Resultados}

Flujos de exergía

Las figuras (5), (6) y (7) muestran el porcentaje de exergía de cada uno de los modos de transporte que ocurren en un destilador solar en comparación con la entrada de exergía. En el caso de las figuras (6) y (7) se muestran dos columnas para cada modo de transporte, debido a que cada una corresponde al valor mínimo y máximo del parámetro de estudio.

La figura 5 muestra los porcentajes de exergía de cada uno de los modos de transporte en el destilador cuando la irradiancia solar tiene un valor de 1,000 W/ $\mathrm{m}^{2}$. Se ha supuesto que cuando la irradiancia solar es nula, no existen flujos de exergía en el destilador (equilibrio térmico con el medio ambiente).

El mayor flujo de exergía está en la interacción colector-agua con cerca de 13\%, mientras que el menor son las pérdidas, a través del aislamiento. Además, como se verá, esta condición $\left(G_{S}=1,000 \mathrm{~W} / \mathrm{m}^{2}\right)$ produce los mayores flujos de exergía en comparación con los demás parámetros.

La figura 6 muestra que cuando la temperatura ambiente varía desde cero hasta los $50^{\circ} \mathrm{C}$, el cambio en los flujos de exergía es marginal y en decremento de los flujos. Se debe recordar que la irradiancia solar de entrada es de $500 \mathrm{~W} / \mathrm{m}^{2}$ de acuerdo con la tabla 1 y el procedimiento del cálculo.

Sin embargo, los resultados experimentales (Morse y Read, 1968), apuntan a que una mayor temperatura ambiente contribuye significativamente a una mayor producción de destilado, debido al incremento en la diferencia de temperaturas entre el agua salada y el vidrio. 


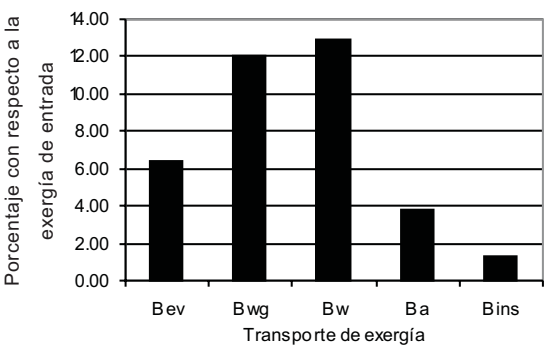

Figura 5. Porcentaje de la exergía total en los diferentes modos de transporte en el destilador solar para irradiancia solar $G S=1,000 \mathrm{~W} / \mathrm{m}^{2}$

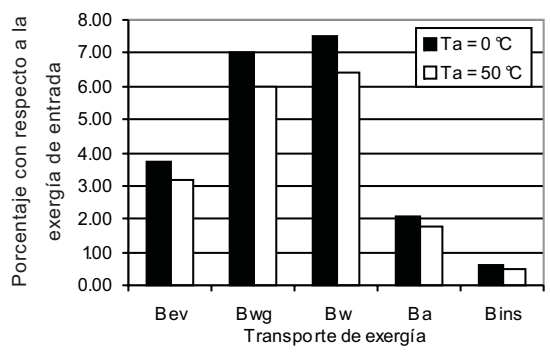

Figura 6. Porcentaje de la exergía total en los diferentes modos de transporte en el destilador solar para temperatura ambiente $T_{a}=0$ y $50{ }^{\circ} \mathrm{C}$.

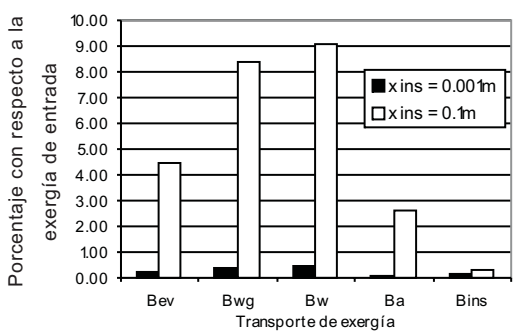

Figura 7. Porcentaje de la exergía total en los diferentes modos de transporte en el destilador solar para temperatura ambiente $x_{i n s}=0.001$ y $0.1 \mathrm{~m}$

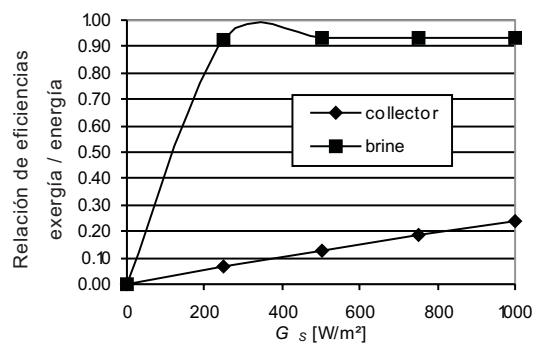

Figura 8. Relación eficiencia exergía/energía $\varphi$, cuando GS varía de 0 a 1,000 W/m² 
Estos autores apuntan a que existe un incremento en los coeficientes de transferencia de calor por convección y de evaporación cuando aumenta la temperatura ambiente. El modelo convencional seguido en este trabajo considera los coeficientes convectivos de transferencia de calor como constantes, por lo que no puede notarse este efecto.

Por otro lado, la figura 7 indica que el aislamiento térmico, cuando los demás parámetros permanecen constantes, es crítico para un buen funcionamiento del destilador en término de la energía y exergía.

Nótese que no hay una reducción drástica en las pérdidas por el aislamiento térmico, como se podría suponer, sino lo contrario. Se observa que todos los flujos de exergía en el destilador se incrementan, sobre todo, la exergía entre el colector y el agua.

Esto quiere decir que el aislamiento propicia que el colector utilice mucha más exergía en su interacción con el agua salada, no sólo por el aumento del flujo de calor sino, y aún más importante, por el aumento de la temperatura del colector.

\section{Eficiencias de energía y exergía}

La relación de eficiencias exergía/energía para el colector y para el agua salada, respectivamente, se definen según:

$$
\begin{gathered}
\varphi_{e v}=\frac{\eta_{e x, e v}}{\eta_{e n, e v}} \\
\varphi_{c o l}=\frac{\eta_{e x, c o l}}{\eta_{e n, c o l}} .
\end{gathered}
$$

Este indicador $\varphi$ muestra la relación que hay entre la calidad y la cantidad de la energía. Se puede mostrar que $\varphi$ está en función únicamente de las temperaturas de los sistemas:

$$
\begin{aligned}
\varphi_{e v} & =\frac{1-T_{a} / T_{w}}{1-T_{a} / T_{c o l}} \\
\varphi_{c o l} & =\frac{1-T_{a} / T_{c o l}}{\left(1+\frac{1}{3}\left(\frac{T_{a}}{T_{c o l}}\right)^{4}-\frac{4 T_{a}}{3 T_{c o l}}\right)} .
\end{aligned}
$$

Así, la relación $\varphi$ es un cociente de dos eficiencias de Carnot, donde dos sistemas están en contacto térmico entre ellos pero ambos se comparan contra un estado de referencia. La figura 8 muestra la relación $\varphi$ cuando la irradiancia solar varía de 0 a $1,000 \mathrm{~W} / \mathrm{m}^{2}$.

La eficiencia exérgica en el colector es, como podríamos haber anticipado, pequeña en comparación con la eficiencia energética. Sin embargo, muestra un aumento considerable hasta más de $20 \%$ cuando la irradiancia solar es máxima.

Es posible mostrar que las irreversibilidades en el colector se incrementan aun cuando la eficiencia de segunda ley aumenta. Esto se debe que el recurso o la exergía de entrada, se incrementa con una tasa mayor que aquélla con la que se producen las irreversibilidades.

Para el agua salada, con un valor reducido de irradiancia solar, $\varphi$ posee valores cercanos a la unidad, lo cual muestra que casi no existen irreversibilidades en el proceso de transporte de calor entre el colector y el agua, es decir, la calidad en el mecanismo de transporte se aprovecha casi al 100\%. Podemos decir que, no existiendo pérdidas de calor en el colector, el sistema colector-evaporador es altamente eficiente.

La temperatura ambiente, como se vio en los resultados de los flujos de exergía, no afecta a las variables de segunda ley. Esto se debe a que, según el modelo, cuando la irradiancia permanece constante, las temperaturas ambiente y de los componentes del destilador varían en la misma proporción, de manera que las diferencias de temperatura permanecen constantes.

Este defecto no se debe al modelo, sino al estudio paramétrico, ya que es bien sabido que la temperatura ambiente está fuertemente relacionada con la irradiancia solar. Sin embargo, en comparación con el cambio en las variables que produce la variación en la irradiancia solar, se puede observar que la temperatura ambiente es un parámetro secundario.

A pesar de que los flujos de exergía muestran un gran incremento cuando el espesor del aislamiento aumenta (figura 7), la relación entre exergía y energía, tanto para el colector como para el agua salada, casi no produce cambios significativos.

El agua salada no modifica esta relación, lo cual significa que ambas eficiencias crecen con la misma tasa, mientras que en el colector se observa un incremento hasta más del 0.14 hasta cierto valor crítico del espesor del aislamiento, aproximadamente igual a $0.01 \mathrm{~m}$. Esto indica que más aislamiento térmico del crítico no tiene un efecto considerable para el almacenamiento de energía térmica en el colector. 


\section{Discusión}

Generalmente se refiere a la exergía o la entropía como propiedades de los sistemas que dan una descripción cuantitativa de la calidad de la energía contenida en el mismo. Así mismo, se dice que las eficiencias de segunda ley son una mejor herramienta para la evaluación de los diseños de equipos, cuyo funcionamiento dependa de variables termodinámicas. En el rubro de la destilación solar, con excepción de un trabajo hecho por Kwatra (1996) que utiliza la exergía para el cálculo de un área de evaporación óptima, no existen análisis de segunda ley. Esto indica, entre muchos otros factores, que la comunidad ingenieril y científica solar, se limita al empleo de la primera ley de la termodinámica para el análisis de los sistemas solares.

Pensamos que una de las causas es que los valores de las eficiencias de segunda ley de los sistemas solares son bastante más bajas en comparación con las eficiencias de primera ley. Los bajos valores de eficiencia suponen un bajo rendimiento con respecto a otras tecnologías y por consiguiente, una pérdida de competitividad en el mercado de los equipos de conversión de energía. Sin embargo, las eficiencias son indicadores de qué tan bien opera un equipo con cierto recurso.

La eficiencia de segunda ley describe un equipo o un proceso en términos de la calidad de la energía contenida en éste. Esta calidad está directamente relacionada con la variable termodinámica intensiva en el análisis. Por ejemplo, la calidad de un sistema será mayor si su valor de temperatura es mayor, así como la calidad de un sistema que consiste en una caída de agua será mayor mientras mayor sea la altura de esta caída. Los flujos de calor $y$, por consiguiente las eficiencias de primera ley, están relacionados con potenciales de temperatura: debe existir un gradiente de temperaturas para que ocurra el flujo. Entre mayor sea el potencial, mayor será el flujo. Si los materiales no varían sus propiedades físicas con la temperatura, el flujo de calor será el mismo a distintas temperaturas pero conservando el mismo gradiente.

La exergía requiere para su definición un estado de referencia. Este estado de referencia indica cuánta utilidad puede aprovecharse del contenido energético del sistema. Así, para una eficiencia de segunda ley, no solo se requiere conocer el gradiente de temperaturas, sino que también la temperatura a la cual ocurre dicha interacción.

El valor de una eficiencia de primera ley puede ser el mismo para evaluar tanto un horno metalúrgico como un refrigerador criógenico, pero no lo es para una eficiencia de segunda ley. Por consiguiente, la exergía constituye un concepto más adecuado para la evaluación termodinámica de equipos y procesos. Los

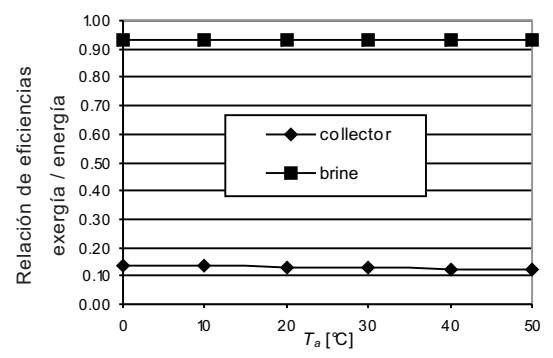

Figura 9. Relación eficiencia exergíalenergía $\varphi$, cuando Ta varía de 0 a $50^{\circ} \mathrm{C}$

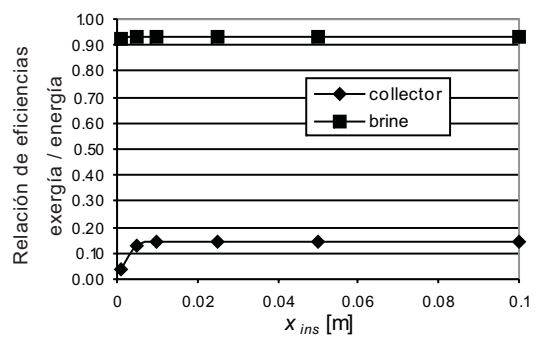

Figura 10. Relación eficiencia exergía/energía $\varphi$, cuando $x_{i n s}$ varía de 0.001 a $0.1 \mathrm{~m}$ 
sistemas de conversión de energía cuya fuente es la radiación solar tienen 2 problemas fundamentales:

1) la naturaleza periódica de la fuente de radiación y

2) la baja eficiencia de los sistemas colectores de la radiación.

La naturaleza periódica de la fuente solar se puede solucionar con equipos de almacenamiento de energía, lo cual, como es bien sabido, incrementa considerablemente el costo de los sistemas solares. Con respecto a la eficiencia de los sistemas solares, se debe especificar cuál eficiencia se toma en cuenta: la eficiencia de primera o de segunda ley. Las eficiencias de primera ley son bajas cuando existen interacciones de masa y energía hacia fuera del sistema, o debido a reacciones químicas incompletas. Las eficiencias de segunda ley son bajas por las mismas razones, y además, cuando existe un recurso en un estado de mucha mayor calidad que el estado al que llega el producto o el efecto útil. Concretamente, en el destilador solar, el colector además de tener pérdidas de energía por radiación y conducción, alcanza temperaturas muy por debajo de la temperatura de la fuente de exergía: la superficie del sol. Sin embargo, un sistema solar no debe ser evaluado de esa manera.

En una planta termoeléctrica, alto horno, ingenio azucarero, etc, las fuentes de exergía, cualquiera que sean, representan un gasto mayor en la operación del proceso: combustóleo, carbón, electricidad, gas natural, biomasa, etc. son recursos que se pueden elegir, manejar e incluso modificar; sin embargo, la radiación solar no. La radiación solar es un recurso gratuito y ciertamente inmodificable (a menos que se utilice concentración solar, lo cual eleva la temperatura del colector solar). Si la eficiencia de una caldera debe tomarse como la exergía del vapor producido en relación con la exergía del combustible, la eficiencia de un destilador solar, al igual que el de cualquier sistema solar debe ser la relación de la exergía del efecto útil (evaporación) entre la exergía que se extrae del colector (interacción colector-evaporador). Así resulta que los sistemas solares son aún más eficientes que otros equipos de conversión de energía. Esta conclusión no implica que no sea válido establecer eficiencias en la interacción sol-colector. Sin embargo, esta eficiencia no debería tomarse en cuenta cuando se compare con otros sistemas de conversión de energía. En el caso del destilador, la eficiencia de segunda ley del agua salada, $\eta_{\text {ex,ev }}$, posee valores bastante altos, debido a que la diferencia de temperaturas entre el colector y la masa de agua es muy pequeña, por lo tanto, no se producen grandes irreversibilidades. En resumen, la fuente de exergía terrestre de recolección solar debe ser tomada como el recurso en los cálculos de eficiencias de segunda ley.

Un destilador solar es un dispositivo de alta eficiencia según la segunda ley de la termodinámica y la discusión de su empleo no debe pasar por argumentos teóricos, sino por viabilidades de material, costos y mantenimiento.

\section{Referencias}

Bejan A. Entropy Generation Through Heat and Fluid Flow. New York. Wiley. 1982.

Bejan A. Unification of Three Different Theories Concerning the Ideal Conversion of Enclosed Radiation. Trans ASME, J. Solar Energy Engineering, 109:46-51. 1987.

Bejan A. Entropy Generation Minimization. The Method of Thermodynamic Optimization of Finite-Size Systems and Finite-Time Processes. New York. CRC Press. 1996.

Bejan A. Advanced Engineering Thermodynamics. New York. Wiley. 1988.

Gaggioli R.A. Available Energy and Exergy. International Journal of Applied Thermodynamics, 1:1-8. 1998.

Hongfei Z., Xiaoyan Z., Jing Z., Yuyuan W. A Group of Improved Heat and Mass Transfer Correlations in Solar Stills. Energy Conversion and Management, 43:2469-2478. 2002.

Kotas T.J. The Exergy Method of Thermal Plant Analysis. London. Butterworths. 1994.

Kumar S., Tiwari G.N. Estimation of Convective Mass Transfer in Solar Distillation Systems. Solar Energy, 57 (6):459-464. 1996.

Kwatra H.S. Performance of a Solar Still: Predicted Effect of Enhanced Evaporation Area on Yield and Evaporation Temperature. International Journal of Solar Energy, 56(3): 261-266. 1996.

Malik M.A.S., Tiwari G.N., Kumar A., Sodha M.S. Solar Distillation: A Practical Study of a Wide Range of Stills and their Optimum Design, Construction and Performance. Oxford. Pergamon Press. 1982.

Moran J.M. Availability Analysis: A Guide to Efficient Energy Use. Corrected Edition. New York. ASME Press. 1989.

Morse R.N, Read W.R. A Rational Basis for the Engineering Development of a Solar Still. Solar Energy, 12:5-17. 1968.

Petela R. Exergy of Heat Radiation. Trans. ASME, J. Heat Transfer, 2:187-192. 1964.

Petela R. Exergy of Undiluted Thermal Radiation. Solar Energy, 74:469-488. 2003.

Porta M.A., Chargoy N., Fernandez-Zayas J.L. Extreme Operating Conditions in Shallow Solar Stills. Solar Energy, 61:465-476. 1997. 
Shukla S.K., Sorayan V.P.S. Thermal Modelling of Solar Stills: an Experimental Validation. Renewable Energy, 30: 689-699. 2005.

Szargut J., Morris D.R., Steward F.R. Exergy Analysis of Thermal, Chemical, \& Metallurgical Processes. New York. Hemisphere Publishing Corporation. 1988.

Tiwari G.N., Singh H.N., Tripathi R. Present Status of Solar Distillation. Solar Energy, 75:367-373. 2003.
Torres-Reyes E., Picón-Núñez M., Cervantes-de Gortari J. Exergy Analysis and Optimization of a Solar-Assisted Heat Pump. Energy Int. Journal, 23:337-344. 1998.

Tripathi R., Tiwari G.N. Effect of Water Depth on Internal Heat and Mass Transfer for Active Solar Distillation. Desalination, 173:187-200. 2005.

\section{Semblanza de los autores}

Juan Cristóbal Torchia-Núñez. Estudió la licenciatura y maestría en ingeniería mecánica en la Facultad de Ingeniería de la UNAM. Actualmente realiza sus estudios de doctorado en ingeniería mecánica en el área de termofluidos y desde hace más de 5 años trabaja con el Dr. Cervantes de Gortari. Es profesor de laboratorio desde hace 4 años en la Facultad de Ingeniería de la UNAM.

Miguel Ángel Porta-Gándara. Es ingeniero en comunicaciones y electrónica por el Instituto Politécnico Nacional, obtuvo el grado de doctor en ingeniería por la Universidad Nacional Autónoma de México. Actualmente se desempeña como investigador en el Centro de Investigaciones Biológicas del Noroeste, S.C.

Jaime G. Cervantes de Gortari. Egresó como ingeniero mecánico electricista por la UNAM en 1970; obtuvo la maestría en ingeniería mecánica por la misma institución en 1972. Asimismo, obtuvo el doctorado (Ph.D.) en ingeniería mecánica en Purdue University, EUA en 1976. Es profesor titular "C" de tiempo completo, definitivo en la Facultad de Ingeniería de la UNAM; miembro del Sistema Nacional de Investigadores nivel III; participa en el Programa de Primas al Desempeño del Personal Académico de Tiempo Completo (PRIDE) con nivel D y fue catedrático UNAM nivel 2 durante el tiempo de este programa. 